\title{
L'angle mort du débat sur la « juste part » dans le conflit étudiant au Québec : les banques. Un conflit idéologique qui ne dit pas son nom
}

Sylvie Paquerot

\section{(2) OpenEdition}

Journals

Édition électronique

URL : http://journals.openedition.org/conflits/18512

DOI : $10.4000 /$ conflits. 18512

ISSN : $1777-5345$

Éditeur :

CCLS - Centre d'études sur les conflits lilberté et sécurité, L'Harmattan

Édition imprimée

Date de publication : 26 décembre 2012

Pagination : 174-180

ISBN : 978-2-336-00248-4

ISSN : 1157-996X

Référence électronique

Sylvie Paquerot, «L'angle mort du débat sur la « juste part » dans le conflit étudiant au Québec : les banques. Un conflit idéologique qui ne dit pas son nom », Cultures \& Conflits [En ligne], 87 | Automne 2012, mis en ligne le 26 décembre 2012, consulté le 30 mars 2021. URL : http://

journals.openedition.org/conflits/18512 ; DOI : https://doi.org/10.4000/conflits.18512 


\section{L'angle mort du débat sur la " juste part » dans le conflit étudiant au Québec : les banques. Un conflit idéologique qui ne dit pas son nom}

\section{Sylvie PAQUEROT}

Sylvie Paquerot est professeure agrégée à l'École d'études politiques de l'Université d'Ottawa (Canada). Elle est également professeure associée au département des sciences juridiques de l'Université du Québec à Montréal (UQAM). À travers ses recherches sur la gouvernance mondiale de l'eau, elle s'intéresse particulièrement aux enjeux de légitimité des décisions à l'échelle internationale et à la fonction politique du droit dans ce contexte. Elle a publié en 2011 L'eau en commun avec Gabriel Blouin-Genest et Frédéric Julien (Québec, PUQ).

$\mathrm{D}$

ès le début de la grève étudiante au Québec, l'argument principal, voire unique, du gouvernement de cette province pour justifier la hausse des frais de scolarité était que les étudiants devaient payer leur « juste part ». Or il faut bien constater, plusieurs mois après le début du conflit, que si elle est inlassablement revenue dans le discours gouvernemental ${ }^{1}$, la formule ne semble pas pour autant avoir suffisamment retenu l'attention des médias pour qu'un véritable débat s'engage sur son sens et la pertinence de son application. Les discours tout azimut sur la « juste part » sans analyse plus approfondie de ce dont il est question semblent d'autant plus surprenants qu'une telle analyse permet selon nous de capturer l'essence même du conflit qui a eu cours : un changement d'orientation de société, de compromis social, sans véritable débat démocratique.

Le problème du sous-financement des universités n'a rien de nouveau au Québec. Celui-ci a été chiffré dès 2003 et une commission parlementaire générale a été constituée en 2004 pour discuter du problème ${ }^{2}$. Cependant, on ne

1. Le ministre Bachand a encore réitéré le 22 juin dernier, lors d'une énième grande manifestation en appui aux étudiantes et étudiants, qu'il n'y a pas lieu de rouvrir quelque négociation que ce soit si ces derniers n'admettent pas d'entrée de jeu le principe de leur contribution au financement des universités. 
retrouve sur le site internet de l'Assemblée nationale du Québec que les résultats d'audiences partielles et non générales sur cette question ${ }^{3}$. C'est que l'identification du problème n'a pas été suivie d'un débat large permettant de confronter les différentes perspectives de financement du réseau universitaire, et donc les différentes visions de la fonction qu'il doit tenir dans la société. Plutôt, le gouvernement Charest a choisi d'ouvrir la discussion sur les équilibres à tenir dans le cadre d'une seule orientation, préalablement déterminée : celle du financement sur le modèle nord-américain, évacuant ainsi, sans plus de discussion, le choix de société effectué quatre décennies plus tôt lors de la vaste réforme du système de l'éducation dont un des objectifs explicites était bien de tendre vers la gratuité scolaire ${ }^{4}$. Or en démocratie, lorsque la conflictualité ne peut plus être encadrée, débattue et résolue dans le cadre des institutions, ne serait-ce que temporairement, elle ne disparaît pas pour autant. Elle se transpose au-delà de ces institutions, se diffuse au sein de la société, ici dans la rue, qui devient le théâtre de la confrontation politique ${ }^{5}$.

\section{Une transformation sous-jacente de la nature et de la fonction de l'insti- tution universitaire}

«C'est la nature même de notre système d'éducation qui est en jeu » a soutenu notamment l'Association pour une solidarité syndicale étudiante (ASSÉ), affirmation qui symbolisait magnifiquement l'incompréhension fondamentale, radicale, entre le gouvernement et les associations étudiantes dans ce conflit. C'est que, du point de vue gouvernemental, une solution collective à un problème d'action collective avait déjà été définie dans le cadre des discussions qui avaient eu lieu avec les dirigeants des institutions universitaires. Le compromis trouvé s'appuyait sur l'équilibre de trois contributeurs principaux : le gouvernement (c'est-à-dire les contribuables), les fonds privés volontaires (par le biais du mécénat ${ }^{6}$ et du financement de la recherche) et les étu-

2. Rappelons ici qu'une autre grève étudiante a eu lieu en 2005 au Québec : Fédérations des associations étudiantes du campus de l'université de Montréal, «Le sous-financement des universités », 9 décembre 2011, www.faecum.qc.ca/sociopolitique/le-sous-financement-desuniversites, page consultée le 25 octobre 2012.

3. Rapport sur les auditions des dirigeants des établissements d'enseignement de niveau universitaire; En application de la Loi sur les établissements d'enseignement de niveau universitaire, rapport et recommandations, février 2011, Assemblée nationale du Québec, ironiquement sous-titré «Place aux citoyens » (www.assnat.qc.ca/fr/travaux-parlementaires/commissions/cce-39-1/index.html\#mandats Termines, consulté le 25 octobre 2012.)

4. Le très célèbre Rapport de la Commission Parent (1965) au Québec, présente les orientations choisies à cette époque par la société québécoise en matière d'éducation, et se montre très explicite quant à la volonté de tendre vers la gratuité, au niveau universitaire, comme le rappelle un membre de cette commission : Rocher G., Perrier Y., « La lutte des étudiants est juste, dit Guy Rocher. L'un des penseurs du système d'éducation québécois prône l'abolition des droits de scolarité ", Le Devoir, 11 avril 2012 (www.ledevoir.com/societe/education/347145/la-lutte-des-etudiants-est-juste-dit-guy-rocher, consulté le 25 octobre 2012).

5. Nous avons ailleurs développé plus largement cette analyse dans un papier présenté au congrès de l'International Political Science Association (IPSA), Madrid, juillet 2012 (www.ipsa.org/sites/default/files/ipsa-events/madrid2012/papers/paper-8666-2012-06-181520.pdf, consulté le 25 octobre 2012)

6. Il est assez ironique de constater que dans cette discussion, les universités ont accepté qu'une 
diants «clients " du service (soit la fameuse «juste part » imposée dans le cadre d'une loi budgétaire). Or, du point de vue des étudiantes et étudiants, non seulement cette "part » était injuste, mais elle contribuait de surcroît à l'alourdissement de l'endettement étudiant, et le compromis trouvé induisait un changement majeur dans la mission même des universités. Injuste, il l'était même dans une perspective marchande ${ }^{7}$ parce que si une personne détentrice d'un diplôme universitaire a globalement plus de chances de trouver un travail stable et rémunérateur, il faut bien être conscient que ce n'est pas vrai pour tous les secteurs. On sait bien qu'un doctorat en philosophie n'a pas la même «valeur marchande » sur le marché du travail qu'une licence en informatique, par exemple. Injuste donc parce que l'on fait peser uniformément le coût sur des « clients» qui ne consomment pas des «biens » équivalents. Il est bien connu d'ailleurs dans le milieu universitaire que les sciences humaines et sociales sont la «vache à lait » des sciences dites dures, dont les coûts de fonctionnement - laboratoires, etc. - sont largement plus élevés. Cette dimension a été rapidement abordée dans l'espace public et certains ont proposé une modulation par secteur de l'augmentation prévue. Injuste ensuite parce qu'un coût à l'entrée peut avoir pour effet d'exclure ceux et celles qui ne disposent pas des sommes nécessaires, en amont de la formation supposée leur permettre des bénéfices futures, ce qui nous amène au deuxième argument, celui de l'endettement. La proposition gouvernementale pour réduire l'effet d'exclusion potentiellement engendré par la hausse des frais imposée reposait en effet, in fine, sur le risque d'une augmentation du fardeau de dettes contractées ${ }^{8}$ par les étudiants. Or, rejoignant l'argument précédent, cet endettement n'aurait pas pesé du même poids sur les revenus futurs selon que l'on aurait choisi une filière universitaire rentable ou non. D'un point de vue strictement marchand, on peut comprendre que ce choix de mode de financement puisse paraître rationnel. S'agissant $\mathrm{du}$ « marché » de la formation et du « marché » du travail, l'objectif est alors de créer des incitations afin que l'offre rencontre la demande sur ces deux marchés et qu'ils s’équilibrent. Si je dois individuellement investir dans mon «capital humain » et que pour ce faire je dois m'endetter, j'aurais alors tout intérêt à choisir une filière de formation dont le marché du travail a fortement besoin, ce qui me garantira un revenu supérieur, à même d'éponger les dettes contractées. Bizarrement, cet aspect de la discussion a été relativement ignoré par la sphère publique alors même que les conséquences de ce modèle institutionnel ailleurs en Amérique du Nord sont largement connues : familles endettées jusqu'à la retraite par les dettes étu-

part de leur financement doive reposer sur les contributions volontaires et aléatoires du mécénat, sans analyse approfondie, là encore, de la portée d'un tel choix dans une petite société comme le Québec où le potentiel de contributeur s'avère relativement réduit.

7. Nous ne voulons en aucun cas laisser entendre ici que ceci représente la position des étudiants. Il s'agit plutôt de discuter de manière plus générale du fondement argumentaire de la «juste part».

8. Bien que le gouvernement ait soutenu qu'il augmenterait le plafond de la part de bourse (non remboursable) en proportion de la hausse des frais, on sait que la part des prêts n'a cessé d'augmenter d'année en année dans les deux dernières décennies. 
diantes des parents et des enfants, offre de crédit bancaire modulée selon le choix de domaine d'études 9 , etc.

Ce qui nous amène au dernier argument, celui du sens même et de la fonction de l'institution universitaire. Nous ne pouvons évidemment reprendre ici tous les termes du débat sur la fonction de l'université dans la société mais, pour comprendre le sens et la portée du conflit étudiant au Québec, il importe tout de même d'en souligner au minimum un aspect central : la double fonction qu'ont développée les universités au Xxe siècle, soit celle de former de la main d'œuvre qualifiée pour le marché du travail, en sus de leur mission première d'être un lieu pour penser, réfléchir, approfondir notre compréhension du monde par la recherche indépendante qui, peu à peu, semble devenue obsolète du point de vue de nos gouvernements, voire même de nos directions universitaires. Marginalisée 10 dans le débat public, inabordable dans le cadre de la conception privilégiée par le gouvernement Charest (le principe de l'utilisateur-payeur), cette dimension en constitue pourtant bien le cœur.

\section{Pour comprendre le conflit : le principe utilisateur-payeur et la concep- tion néolibérale du juste 11}

Il faut en effet replacer le récent conflit étudiant dans le contexte québécois plus large d'un gouvernement qui, dès son arrivée au pouvoir, avait explicitement entrepris de remettre en cause le modèle de société mis en place au Québec, fondé sur une large inclusion citoyenne, d'importants services publics 12 - avec pour corollaire un taux d'imposition plus élevé que dans les autres provinces canadiennes ou aux États-Unis - mais des inégalités moins grandes 13. La compétitivité du Québec en Amérique du Nord a bien été le mantra de ce gouvernement, dans tous les domaines : taux d'imposition bien sûr, mais également tarifs hydroélectriques préférentiels pour les entreprises, redevances sur les ressources naturelles au plus bas, voire exemption, réduction des contraintes environnementales, etc. De budget en budget, pendant neuf ans, ce gouvernement n'a eu de cesse d'élargir les domaines d'implantation d'une logique marchande, soit en privatisant certaines fonctions aupara-

9. Enseignant à l'université d'Ottawa, nos propres étudiants connaissent bien le principe selon lequel ils auront accès à un crédit bancaire plus élevé en s'inscrivant en droit plutôt qu'en sciences sociales par exemple. Or, même ces filières ne mènent pas nécessairement à un emploi garanti!

10. Bien que certains acteurs du conflit l'aient mise en débat, peu de médias l'ont relayé. La revendication des associations étudiantes pour des états généraux de l'éducation se fondait précisément sur ce constat.

11. Un intéressant pamphlet publié au Québec par deux professeurs de philosophie fournit des clés de raisonnement pertinentes pour réfléchir à la question du juste proposée par l'idéologie néolibérale et à l'enjeu de l'action collective qu'elle oblitère : Robichaud D. et Turmel P., La juste part : repenser les inégalités, la richesse et la fabrication des grille-pain, Montréal, Atelier 10, 2012.

12. En contexte nord-américain. Les services publics au Québec ne sont pas plus étendus que dans la majorité des pays européens.

13. Robichaud et Turmel, op. cit., p. 81. 
vant exercées par l'État, soit en instaurant des partenariats public/privé, soit encore en introduisant un principe de paiement direct individualisé pour l'utilisation des services dits publics. Chacun devait être responsable de ce qu'il utilise ou consomme, transformant peu à peu les citoyens en consommateurs. Et c'est bien là qu'il faut situer l'ampleur qu'a pris le conflit étudiant : le principe d'utilisateur-payeur pour les services publics que souhaitait largement implanter le gouvernement Charest, ce dernier l'a testé d'abord auprès des universités, où, pouvait-il croire, le renversement du choix de société serait moins perceptible car un système de frais préexistait. Ce n'est cependant pas ce qui s'est produit et une part significative de la population l'a bien compris. Or, même en acceptant l'idée que la formation universitaire menant à des emplois bien rémunérés devait être en partie prise en charge par ceux et celles qui la «consomment » en tant qu'investissement dans leur propre capital humain, la question de savoir qui doit payer pour la dimension citoyenne et collective de la mission universitaire - penser notre monde - demeure entière.

\section{Un débat soigneusement évité : Qu'est-ce qui est juste au fait ? Selon quels critères ?}

Nous discutons depuis plusieurs mois maintenant de l'université. Or qu'est-ce que l'université ? Pourquoi la considère-t-on plus ou moins comme un service public ? Pourquoi débattons-nous de « juste part » sans que cela renvoie automatiquement au «prix que cela coûte » comme une chaise ou une conserve de petits pois ? C’est que, en premier lieu, aucune société ne se porterait bien, comme société, si aucun jeune n'allait à l'université : c'est donc dire que l'université, la formation universitaire, n'est pas un bien de consommation comme un autre ${ }^{14}$. Elle est un bien public au sens où il y a un bénéfice pour la société tout entière à ce que plus de jeunes choisissent d'aller à l'université ${ }^{15}$, de retarder ainsi le moment où il « rapportera de l'argent ", où il pourra financièrement répondre à ses propres besoins et sera considéré par la société comme un «producteur»16. C'est aussi que, selon les statistiques, les jeunes qui poursuivent leurs études ont plus de chances d'avoir de meilleurs revenus et une meilleure sécurité/stabilité par la suite, ce qui constitue un bien à la fois pour l'individu et pour la collectivité puisqu'il paiera plus d'impôts, mais également parce que des personnes en situation de meilleure sécurité/stabilité induisent moins de coûts sociaux ${ }^{17}$. Il y a donc ici deux parties qui tirent bénéfice, collectivement - la société - et individuellement - l'étudiant - de

14. Même pour Adam Smith, dans De la richesse des nations, l'éducation relève du souverain, précisément parce qu'elle est nécessaire à la société alors même qu'il n'est pas profitable individuellement d'y investir ce que cela coûte, comme toutes les grandes infrastructures.

15. Rappelons ici que la société québécoise se situe encore en-dessous de la moyenne canadienne s'agissant du taux de diplômes universitaires dans la population.

16. Il me faut dire ici qu'en tant que professeure, je n'ai jamais trouvé idéale la situation dans laquelle nous sommes où de plus en plus d'étudiants doivent travailler plus de 10 ou 15 heures par semaine en poursuivant leur scolarité : la plupart de mes collègues vous confirmeraient sans doute que ce n'est pas sans effet d'une part sur leurs résultats et d'autre part sur leur intérêt à poursuivre leurs études. 
l'existence de l'université et de sa qualité. C'est dans ce contexte qu'il faut replacer ce que sont les justes parts des uns et des autres. On peut donc dire que la société, collectivement, et les étudiants, individuellement, tirent bénéfice de leur éducation universitaire sur le long terme (en considérant cependant que cela ne rime pas toujours avec de hauts salaires ensuite). Il serait donc " juste » qu'à la fois la société, par l'impôt, et les étudiantes et étudiants, contribuent. Dans le débat toutefois, bien que l'idée ait été émise que la « juste part » des étudiants doive être plus justement prélevée, en aval plutôt qu'en amont, à travers des taux d'imposition reflétant mieux l'avantage effectif qu'ils tirent de leur formation universitaire, elle n'a pas semblé recueillir beaucoup d'adhésion ${ }^{18}$. En revanche, il y a un autre segment de la société qui lui récolte des bénéfices du système que nous avons collectivement mis sur pied sous forme de profits à court terme et tout le temps, sans aucun risque : les banques. Autrement dit, l'action collective générée par l'identification du problème de l'accès à l'université a généré une solution qui rencontre en partie ses objectifs - un accès élargi à l'éducation supérieure par la mise sur pied d'un système de prêts et bourses dont les premiers sont garantis par l'État - mais qui, de manière collatérale, a produit également un bénéfice non escompté pour un acteur spécifique.

Les banques en effet, à travers le système des prêts et bourses ${ }^{19}$ sont assurées d'un bénéfice puisque les prêts sont garantis par le gouvernement. Elles sont donc les seules, ici, à tirer plus de bénéfices qu'elles n'investissent puisqu'elles ne paient rien de spécifique pour cette opportunité de profit que leur fournit notre société à travers leur impôt. Or, même si elles payaient leur juste part d'impôt - ce dont il convient de douter sérieusement - cela ne serait pas « juste » pour autant puisqu'elles tirent un profit auquel les autres contribuables n'ont pas accès. Leur part régulière d'impôt contribue au système universitaire dont elles bénéficient tout autant que le reste de la société : elles peuvent embaucher des personnes plus formées; elles opèrent dans une société plus stable avec moins de problèmes sociaux, etc. $\mathrm{La}$ « juste part » que cherchait désespérément dans les poches des étudiants le gouvernement Charest, c'est dans les poches des banquiers qu'elle se trouve, car les étudiants d'aujourd'hui, une fois leurs études achevées, paieront comme contribuables leur juste part... d'impôt, si tant est que notre système d'imposition soit lui-même juste.

La jeunesse a donc eu raison du point de vue à la fois de la justice et de l'efficacité : la gratuité scolaire jusqu'à l'université est le choix le plus juste qu'une

17. Robichaud et Turmel, op. cit., chapitre 15 : «Pourquoi l'égalité profite à tout le monde », p. 83 et ss.

18. Référons encore une fois le lecteur à Robichaud et Turmel qui montrent bien dans ce même chapitre 15 (1) le recul de la progressivité des paliers d'imposition au Canada et au Québec et (2) le paradoxe de l'appui des classes moyennes à des mesures qui les désavantagent.

19. Notons que les prêts sont largement supérieurs aux bourses octroyées, de nombreux étudiantes et étudiants ne peuvent obtenir de bourses. 
société puisse faire, et nous en avons les moyens. La gratuité scolaire demeure par ailleurs le moyen le plus efficace pour assurer que l'université puisse remplir ses deux fonctions sans qu'elles se nuisent l'une à l'autre... Encore faut-il que l'on puisse aussi considérer comme « juste » que ceux qui s'enrichissent avec l'endettement étudiant paient, eux, leur « juste part »... Le Québec, malheureusement, est singulièrement en manque de «capitalistes éclairés », ce qui explique peut-être en partie la polarisation sociale à laquelle nous avons assisté. 